\title{
The Risk of Esophageal Food Impaction In Eosinophilic Esophagitis Patients; The Role of Clinical And Socioeconomic Factors
}

Tarik Alhmoud ( $\nabla$ tarik.alhmoudmd@promedica.org )

Toledo Hospital

Sami Ghazaleh

University of Toledo Medical Center

Marcel Ghanim

University of Toledo Medical Center

Roberta Redfern

Toledo Hospital

\section{Research Article}

Keywords: Eosinophilic esophagitis, food impaction, gastroesophageal reflux disease, linear furrows, felinization

Posted Date: January 3rd, 2022

DOI: https://doi.org/10.21203/rs.3.rs-1135957/v1

License: (9) This work is licensed under a Creative Commons Attribution 4.0 International License. Read Full License 


\section{Abstract}

Background: Eosinophilic esophagitis (EoE) patients present with dysphagia and often suffer from esophageal food impaction (EFI). EFI can lead to life-threatening perforation, and requires emergent endoscopic intervention. The aim of this study is to evaluate the risk factors for EFI in EoE patients.

Methods: This is a retrospective study performed at a tertiary health care system. Medical records and endoscopy images of EoE cases were reviewed. Clinical characteristics and outcomes including EFIs were documented. We used Zip-code median household income as a surrogate for patients' socioeconomic status.

Results: 291 EoE cases were included, mean age was 42 years. Most patients (65\%) had classic EoE endoscopic findings including linear furrows and/or concentric rings, however, a significant proportion (47\%) had findings suggestive of gastroesophageal reflux disease (GERD), such as the presence of erosive-esophagitis, a hiatal hernia or Schatzki's ring. 48 patients $(16 \%)$ developed one or more esophageal food impaction (EFI). The risk of EFI was less likely in the absence of furrows and/or rings; odds ratio $(\mathrm{OR})=.28,95 \% \mathrm{Cl}(0.11,0.72)[\mathrm{P}=.008]$. Females had less $\mathrm{EFI}$ risk; $\mathrm{OR}=0.42,95 \% \mathrm{Cl}(0.19$, $0.95)[P=.04]$. The type of medical insurance and socioeconomic status was not associated with EFI risk.

Conclusion: EFI risk is higher in EoE patients with esophageal furrows and/or rings and in men. Aggressive treatment might be required in this population. GERD and EoE can coexist in many patients. Further studies are required to examine the role of the socioeconomic status in EoE complications.

\section{Background}

Eosinophilic esophagitis (EoE) is an immune-mediated esophageal disease characterized by eosinophilpredominant inflammation and symptoms of esophageal dysfunction ${ }^{1}$; the disease is mediated by type 2 helper T cells (Th2 cells) and triggered by food antigens. ${ }^{2}$ Patients usually have concurrent atopic conditions such as asthma, eczema or allergic rhinitis.

Individual EoE case reports date back to the early $1980 \mathrm{~s}^{3}$, subsequently case series from the early $1990 \mathrm{~s}$ described EoE as a distinct disease entity in patients with dysphagia and eosinophil-predominant eosinophilia. 4,5 The number of diagnosed cases of EoE has grown exponentially over the last two decades. ${ }^{6}$ Currently the prevalence in adults is 40 to 90 cases per 100000 persons in Europe and North America. 6,7

Patients typically present with solid food dysphagia or less commonly esophageal food impaction (EFI). ${ }^{8}$ Other symptoms of esophageal dysfunction might be present such as odynophagia, chest pain, nausea, vomiting, epigastric pain or gastroesophageal reflux disease (GERD) symptoms of heartburn and/or food regurgitation. $^{2}$ 
According to the most recent guidelines ${ }^{9}$ EoE is diagnosed as the presence of at least 15 eosinophils per high power field (HPF) on esophageal mucosal biopsies, in addition to symptoms of esophageal dysfunction, and the exclusion of other disease conditions that can lead to esophageal eosinophilia (such as pill-induced esophagitis and Crohn's disease). EoE can lead to severe esophageal inflammation and progressive fibrosis and narrowing of the esophageal lumen in some patients. EoE patients may have progressive dysphagia and food impactions that require urgent endoscopic intervention in most cases, and can lead to life threatening esophageal perforation. ${ }^{10}$ We studied our large cohort of EoE patients to identify the risk factors associated with esophageal food impaction. In addition to demographic and clinical factors, this study will also examine socioeconomic status of patients.

\section{Methods}

This is a retrospective cohort study performed at a large Health Care System in northwest Ohio and southeast Michigan, USA. We screened patients with the international classification of disease ICD-9 and/or ICD-10 diagnosis of eosinophilic esophagitis (International Classification of Diseases, Ninth Revision, code 530.13; International Classification of Diseases, Tenth Revision, code K20.0), between years 2007 to 2019. All methods were performed in accordance with the Declaration of Helsinki. Promedica Institutional Review Board (IRB) approval was obtained prior to data collection; written informed consent was waived due to the retrospective nature of the study.

The following inclusion criteria were used:

- Availability of Esophagogastroduodenoscopy (EGD) procedure report and esophageal biopsy results.

- Esophageal mucosal eosinophil count $>=15$ eosinophils per high power field.

- The presence of esophageal dysfunction symptoms including include dysphagia, odynophagia, food impaction, chest pain, epigastric pain, nausea, vomiting, heartburn and regurgitation.

- All other causes of eosinophilic eosinophilia were excluded.

The electronic medical record of each subject was reviewed by one member of the study team and clinical data were manually abstracted. The EGD report and images were reviewed by two researchers including one gastroenterology specialist to verify and document relevant imaging and procedure report findings.

The primary outcome measure of the study was the occurrence of one or more esophageal food impaction (EFI) event requiring endoscopic bolus dislodgement. Logistic regression analysis was used to determine independent predictors of EFI. The model was adjusted for age at diagnosis, sex, ethnicity, body mass index (BMI), socioeconomic status, length of follow up and the presence of EoE findings on endoscopy (defined as the presence of longitudinal furrows and/or diffuse rings (felinization of the esophagus), and the presence of GERD findings on endoscopy (including erosive esophagitis Los Angeles Grade B or greater, hiatal hernia, or Schatzki's ring). 
To asses socioeconomic status we used neighborhood income level as a surrogate. Subjects' zip codes were collected to predict and categorize household income, using the following formula: Median State Adjusted Gross Income (AGI) - Median Zip code AGI (of the year 2004).

The results were categorized into five 'zones' as described below:

- Zone 1: Income level of 0-20th percentile (lowest zip code income compared to state median group)

- Zone 2: income level of 21 st to 40 th percentile

- Zone 3: income level of 41 st to 60th percentile

- Zone 4: income level of 61 st to 80th percentile

- Zone 5: income level of 81st to 100th percentile (highest zip code income compared to state median group)

Continuous variables were reported in two formats: mean \pm standard deviation (SD) or median \pm interquartile range IQR; for variables that are not normally distributed. Chi-square test was performed to test distribution of categorical variables. Two-tailed $p$-value $<0.05$ was considered significant. All statistical analyses were performed using SAS version 9.4 (SAS Institute Inc., Cary, NC, USA).

\section{Results}

\section{Patient Characteristics}

In total, 291 patients met the inclusion criteria for eosinophilic esophagitis diagnosis; a flowchart describing patient exclusions is shown in Figure 1. Detailed chart review and verification of endoscopic images was completed for all subjects who met inclusion criteria. Patients were mainly Caucasian men with a mean age of 42 years. Most patients (75\%) had dysphagia as the presenting symptom. Less than one quarter had gastroesophageal reflux disease (GERD) as the presenting symptom and two percent presented with various other esophageal symptoms. A minority of patients $(16 \%)$ had food impaction as the presenting symptom or later during the disease course (as detailed in patient outcomes section below). Dilation was performed in about half of patients with Bougie, Savary, or Balloon dilation. A detailed description of baseline patient characteristics on study entry is presented in Table 1. 
Table 1

Clinical characteristics of eosinophilic esophagitis cases.

\begin{tabular}{|c|c|}
\hline Age, mean $\pm S D *, n=291$ & $41.8 \pm 13.1$ \\
\hline Sex & 114(39) \\
\hline $\mathrm{F}, \mathrm{n}(\%)$ & $1.6: 1$ \\
\hline \multicolumn{2}{|l|}{ Male to female ratio ( $\mathrm{M}: \mathrm{F}$ ratio) } \\
\hline \multicolumn{2}{|l|}{ Ethnicity, n(\%) } \\
\hline Caucasian & $275(95)$ \\
\hline African American & $9(3)$ \\
\hline Hispanic & $4(1)$ \\
\hline Asian & $1(1)$ \\
\hline $\mathrm{BM}{ }^{\star *}$, mean $\pm \mathrm{SD}, \mathrm{n}=289$ & $30.1 \pm 6.5$ \\
\hline \multicolumn{2}{|l|}{ Medical Insurance, n(\%) } \\
\hline Commercial & $250(86)$ \\
\hline Medicaid & $8(3)$ \\
\hline Medicare & $26(9)$ \\
\hline Self-pay & $7(2)$ \\
\hline Visit, median $\pm I Q R^{\star \star \star}$ & $1 \pm 2$ \\
\hline Length of follow-up (in years) $\pm I Q R$ & $1 \pm 3$ \\
\hline \multicolumn{2}{|l|}{ Indication for EGD, n(\%) } \\
\hline Dysphagia & $178(61)$ \\
\hline Dysphagia with GERD & $41(14)$ \\
\hline GERD without dysphagia & $66(23)$ \\
\hline Other esophageal symptoms: & $6(2)$ \\
\hline \multicolumn{2}{|l|}{ Chest pain, epigastric pain, } \\
\hline \multicolumn{2}{|l|}{ odynophagia, nausea and } \\
\hline \multicolumn{2}{|l|}{ gastrointestinal bleeding } \\
\hline Number of EGDs $s^{\star \star \star *}$, mean \pm SD & $1.7 \pm 1$ \\
\hline Esophageal dilation, n(\%) & $143(49)$ \\
\hline
\end{tabular}

*SD: Standard deviation, ${ }^{* * B M I}$ : Body mass index, ${ }^{\star \star *}$ IQR: Inter-quartile range, ${ }^{* \star \star * E G D:}$ 


\begin{tabular}{|c|c|}
\hline Age, mean $\pm S D *, n=291$ & $41.8 \pm 13.1$ \\
\hline Eosinophil count $\pm S D, n=284$ & $40.6 \pm 26$ \\
\hline Food impaction, $\mathrm{n}(\%)$ & $48(16)$ \\
\hline Perforation, $\mathrm{n}(\%)$ & $1(0.3)$ \\
\hline \multicolumn{2}{|c|}{ *SD: Standard deviation, $\star \star$ BMI: Body mass index, ${ }^{* \star \star}$ QQR: Inter-quartile range, ${ }^{* \star \star \star E G D: ~}$} \\
\hline
\end{tabular}

The mean eosinophil count per HPF per patient was $40.6 \pm 26$ on mucosal biopsies. Two thirds of patients had endoscopic findings classic for eosinophilic esophagitis including longitudinal linear furrows and diffuse concentric rings. Endoscopic findings suggestive of GERD including the presence of hiatal hernia, Schatzki's ring or reflux esophagitis [Los Angeles Classification (LA Class) B, C or D esophagitis] were documented; LA Class A esophagitis was not used as the changes are minor and can be difficult to verify in a retrospective design. At least one GERD-related finding was present in $47 \%$ of patients, mostly hiatal hernia and less frequently Schatzki's ring or reflux esophagitis. A minority of patients (15\%) had normal endoscopic appearance of the esophagus (Figure 2A).

EoE patients had different patterns of esophageal involvement. The most common pattern was the presence of EoE findings (furrows and/or rings only), followed by mixed pattern of EoE findings along with GERD-related findings. A minority of patients had normal endoscopic appearance of the esophagus and the only abnormality was mucosal eosinophilia on biopsy. Mosaic plot of different patterns is shown in Figure 2B. The mosaic plot reveals that one third of patients who lack esophageal EoE findings are more likely to have GERD-related findings.

\section{Patient outcomes}

Esophageal food impaction (EFI) occurred in 48 patients (16\%), 46 of these had EFI at presentation and only two patients $(.007 \%)$ had EFI later during the disease course (both of these patients didn't receive steroid therapy). Nine patients (3\%) experienced multiple food impactions during the study period (mainly due to lack of follow up and/or no use of steroids, and in two cases due to refractory disease).

Esophageal perforation occurred in one patient. Food impactions happened mostly in patients who had endoscopic findings of furrows and/or rings (Figure 3A). The occurrence of food impaction was also investigated as a function of estimated socioeconomic status of patients, where subjects were divided into five groups based on the neighborhood AGI. Patient residences represented 115 unique zip codes in 9 states. Zone 1 included the lowest income group and zone 5 was the highest income compared to median state AGI (as detailed in the methods section). The risk of food impaction did not vary across different incomes categories (Figure 3B).

A logistic regression model was used to determine predictors of the primary outcome of esophageal food impaction (EFI): Females were less likely to develop EFI, odds ratio: $0.424,95 \% \mathrm{Cl}(0.189,0.951)[\mathrm{P}=.04]$. Fewer cases of EFI occurred in the absence of furrows and/or rings on endoscopy, odds ratio: 0.280, 
$95 \% \mathrm{Cl}(0.109,0.718)[\mathrm{P}=.008]$. The model revealed no significant association between the occurrence of $\mathrm{EFI}$ and the following variables: age at diagnosis, type of medical insurance, neighborhood income level, body mass index, eosinophil count on biopsies, length of follow up and the presence of GERD-related endoscopic findings.

\section{Discussion}

In our eosinophilic esophagitis (EoE) patient cohort, 48 cases (16\%) had one or more esophageal food impactions (EFIs); male gender and the presence of classic EoE findings on endoscopy (longitudinal furrows and/or concentric rings in the esophagus) were associated with EFI. EFI is an indicator of severe fibrostenosing EoE or untreated disease, and can lead to serious life-threatening perforation. ${ }^{11}$ Our results are consistent with a large cohort study showing that males are more likely to have esophageal stenosis and food impaction events. ${ }^{12}$ Our results indicate that the presence of furrows and/or rings in EoE patients is a strong predictor of esophageal food impaction and this population should be treated more aggressively to prevent complications. Our findings are consistent with a pediatric study showing that the presence of furrows is associated with food impaction, ${ }^{13}$ no similar study was found in adult population.

Socioeconomic status (SES) of patients affects access to health care, and environmental exposures such as housing and pollution, in addition to food cost and accessibility. Asthma patients with lower SES have increased disease severity and hospitalizations. ${ }^{14,15}$ There are limited data on the role of SES in EoE patient outcomes. A study by Jensen et.al ${ }^{16}$ reviewed the role of different environmental factors in EoE and concluded that EoE might be higher in rural areas. Corder et al described association between EoE diagnosis and houses of brick exterior, forced air or gas heating. ${ }^{17}$ We examined the role of medical insurance and socioeconomic status using patients' neighborhood adjusted gross income as a surrogate of household income. There was no difference in EFI risk across five different levels of household income. Furthermore, neighborhood income was grouped into two categories (high income versus low income) and there was still no difference in the risk of EFI (Supplementary Figure 1). We are the first study (to our knowledge) to examine the role of socioeconomic status in EoE outcomes.

All endoscopy images and patient pathology results of a cohort of 291 patients were reviewed in the current study. Most of the patients in our cohort $(66 \%)$ had the classic EoE findings of esophageal furrows and/or concentric rings, which is higher than what was reported in a large systematic review and meta-analysis study of endoscopic findings in EoE patients that included 4678 patients. ${ }^{18}$ In the systematic review by Kim et al., the prevalence of linear furrows and concentric rings were $48 \%$ and $44 \%$, respectively. ${ }^{18} 17 \%$ of EoE patients in the Kim et. al study had normal endoscopic exam compared to $15 \%$ of patients in our cohort, this emphasizes the importance of obtaining esophageal biopsies in suspected EoE cases, despite normal endoscopic appearance of the esophagus.

It is known that GERD is a leading cause for EFI. ${ }^{19}$ About half of our patients had endoscopic findings suggestive of gastroesophageal reflux disease (GERD), including the presence of a hiatal hernia, erosive 
esophagitis or a Schatzki's ring. Data on the presence of hiatal hernia or Schatzki's ring in EoE patients is limited in the medical literature. A previous study showed that $10 \%$ of patients with Schatzki's rings had EoE, ${ }^{20}$ and a radiology study showed an association between EoE with GERD and Schatzki's rings. ${ }^{21}$ In our cohort, $35 \%$ of patients had hiatal hernia and $24 \%$ had Schatzki's ring. The large systematic review and meta-analysis study of endoscopic findings in EoE patients by Kim et.al ${ }^{18}$ reported that $17 \%$ of EoE patients had evidence of erosive esophagitis compared to $11 \%$ of patients in our study that had LA Class $B$ erosive esophagitis or greater. The lower prevalence of erosive esophagitis in our study may be attributed to the exclusion of patients with LA Class A esophagitis.

GERD is a very common disease with a prevalence of 18 to $28 \%$ in North America. ${ }^{22}$ The fact that GERD can lead to esophageal eosinophilia has impacted the diagnostic criteria of EoE and evolved the way EoE is diagnosed over time. ${ }^{8} \mathrm{~A}$ subset of EoE patients may have complete resolution of esophageal inflammation after treatment with acid suppression medications (proton pump inhibitors (PPIs)). ${ }^{9}$ These patients were previously labelled PPI-responsive esophageal eosinophilia (PPI-REE). Older guidelines (from 2011) recommended treatment with PPIs for 8 weeks to rule out PPI-REE before making the diagnosis of EoE. ${ }^{9}$ However, more recent 2018 guidelines confirmed that PPI-REE is a subtype of EoE and is not a subset of GERD. 8,10,23 In our study, we used the updated EoE guidelines and did not exclude PPIREE cases.

The differentiation between GERD and EoE in patients with esophageal eosinophilia is challenging. It is currently believed that EoE and GERD can coexist in many patients and possibly one condition can trigger the other. ${ }^{9}$ We showed that GERD and EoE coexistence may be more common than previously thought, given that half of our cohort had endoscopic findings suggestive of GERD. The mosaic pattern (Figure 2) clearly shows the different combinations of EoE and GERD endoscopic findings and the difficulty in drawing a line separating these conditions. Furthermore, Figure 2B shows that patients without EoE classic endoscopic findings (the left side of Figure 2B) are slightly more likely to have GERD-related findings than patients who have EoE classic endoscopic findings (the right side of Figure 2B); suggesting that some of our patients might have GERD with increased esophageal eosinophils [esophageal eosinophilia (EE)]; in some cases it is impossible to differentiate GERD with EE from EoE with coexisting GERD.

This study has a number of strengths; we included a relatively large number of patients, and all endoscopic images were reviewed by an expert gastroenterologist. In addition, the availability of subjects' mailing addresses allowed us to investigate socioeconomic status. Our study also has a number of limitations. It is a retrospective study, and as such is subject the inherent limitations of a retrospective design. Some relevant information such as tobacco and ethanol use could not be reliably retrieved. Additionally, patients did not receive standard therapy for EoE, as EoE treatment was evolving over the relatively long timeframe represented in this study, and because patients were treated by multiple gastroenterology specialists. $49 \%$ of our patient underwent esophageal dilation, however, the presence 
edema, strictures and severity of strictures was not consistently documented in our cohort and the Eosinophilic Esophagitis Reference Score (EREFs) couldn't not be calculated.

\section{Conclusion}

EoE patients who have esophageal furrows and/or rings have higher risk of food impaction and probably require more aggressive treatment. GERD and EoE can coexist in many patients and probably more than what was previously thought. Further studies about housing, access to medical care, and the role of socioeconomic status is required. Moreover, future directions include the need for biomarkers to predict the risk of esophageal food impaction as well as a clinical scoring system to predict negative patient outcomes.

\section{List Of Abbreviations}

Adjusted gross income (AGI), body mass index (BMI), eosinophilic esophagitis (EoE), esophageal food impaction (EFI), esophagogastroduodenoscopy (EGD), gastroesophageal reflux disease (GERD), high power field (HPF), interquartile range (IQR), proton pump inhibitor (PPI), PPI-responsive esophageal eosinophilia (PPI-REE), socioeconomic status (SES), standard deviation (SD), type 2 helper T cells (Th2 cells).

\section{Declarations}

- Ethics approval and consent to participate: Promedica Institutional Review Board (IRB) approved this study and approved all experimental protocols. Informed consent waiver was obtained from the Promedica Institutional Review Board due to the retrospective nature of the study. All methods were performed in accordance with Declaration of Helsinki.

- Consent for publication: NA

- Availability of data and materials: All raw data generated or analyzed during this study are included in this published article and its supplementary information files.

- Competing interests: Authors have no conflict of interest to disclose.

- Funding: No funding was provided.

- Authors' contributions: TA: study design, data collection, data analysis and interpretation, and manuscript writing. SG: data collection and analysis. MG: data collection and analysis. RR: study design, manuscript editing.

- Acknowledgements: NA. 
- Authors' information: Tarik Alhmoud is a Gastroenterology and Hepatology Specialist at Promedica Digestive Health Care, and Clinical Assistant Professor, Department of Gastroenterology and Hepatology at the University of Toledo, Toledo, USA.

\section{References}

1. Liacouras CA, Furuta GT, Hirano I, et al. Eosinophilic esophagitis: updated consensus recommendations for children and adults. J Allergy Clin Immunol. 2011;128(1):3-20.e26; quiz 21-22.

2. Furuta GT, Katzka DA. Eosinophilic Esophagitis. N Engl J Med. 2015;373(17):1640-1648.

3. Picus D, Frank PH. Eosinophilic esophagitis. AJR Am J Roentgenol. 1981;136(5):1001-1003.

4. Attwood SE, Smyrk TC, Demeester TR, et al. Esophageal eosinophilia with dysphagia. A distinct clinicopathologic syndrome. Dig Dis Sci. 1993;38(1):109-116.

5. Straumann A, Spichtin HP, Bernoulli R, et al. [Idiopathic eosinophilic esophagitis: a frequently overlooked disease with typical clinical aspects and discrete endoscopic findings]. Schweiz Med Wochenschr. 1994;124(33):1419-1429.

6. Navarro P, Arias A, Arias-Gonzalez L, et al. Systematic review with meta-analysis: the growing incidence and prevalence of eosinophilic oesophagitis in children and adults in population-based studies. Aliment Pharmacol Ther. 2019;49(9):1116-1125.

7. Dellon ES. Epidemiology of eosinophilic esophagitis. Gastroenterol Clin North Am. 2014;43(2):201218.

8. Leigh LY, Spergel JM. An in-depth characterization of a large cohort of adult patients with eosinophilic esophagitis. Ann Allergy Asthma Immunol. 2019;122(1):65-72.e61.

9. Dellon ES, Liacouras CA, Molina-Infante J, et al. Updated International Consensus Diagnostic Criteria for Eosinophilic Esophagitis: Proceedings of the AGREE Conference. Gastroenterology. 2018;155(4):1022-1033.e1010.

10. Schupack DA, Lenz CJ, Geno DM, et al. The evolution of treatment and complications of esophageal food impaction. United European Gastroenterol J. 2019;7(4):548-556.

11. Runge TM, Eluri S, Cotton CC, et al. Causes and Outcomes of Esophageal Perforation in Eosinophilic Esophagitis. J Clin Gastroenterol. 2017;51(9):805-813.

12. Warners MJ, Oude Nijhuis RAB, de Wijkerslooth LRH, et al. The natural course of eosinophilic esophagitis and long-term consequences of undiagnosed disease in a large cohort. The American journal of gastroenterology. 2018;113(6):836-844.

13. Ettyreddy AR, Sink JR, Georg MW, et al. Association between Eosinophilic Esophagitis and Esophageal Food Impaction in the Pediatric Population. Otolaryngol Head Neck Surg. 2018;159(4):750-754.

14. Sahni S, Talwar A, Khanijo S, et al. Socioeconomic status and its relationship to chronic respiratory disease. Advances in respiratory medicine. 2017;85(2):97-108. 
15. Bjur KA, Wi Cl, Ryu E, et al. Socioeconomic Status, Race/Ethnicity, and Health Disparities in Children and Adolescents in a Mixed Rural-Urban Community-Olmsted County, Minnesota. Mayo Clinic proceedings. 2019;94(1):44-53.

16. Jensen ET, Dellon ES. Environmental factors and eosinophilic esophagitis. The Journal of allergy and clinical immunology. 2018;142(1):32-40.

17. Corder SR, Tappata M, Shaheen O, et al. Relationship Between Housing Components and Development of Eosinophilic Esophagitis. Dig Dis Sci. 2020.

18. Kim HP, Vance RB, Shaheen NJ, et al. The prevalence and diagnostic utility of endoscopic features of eosinophilic esophagitis: a meta-analysis. Clin Gastroenterol Hepatol. 2012;10(9):988-996.e985.

19. Lenz CJ, Leggett C, Katzka DA, et al. Food impaction: etiology over 35 years and association with eosinophilic esophagitis. Dis Esophagus. 2019;32(4).

20. Müller M, Eckardt AJ, Fisseler-Eckhoff A, et al. Endoscopic findings in patients with Schatzki rings: evidence for an association with eosinophilic esophagitis. World J Gastroenterol. 2012;18(47):69606966.

21. Towbin AJ, Diniz LO. Schatzki ring in pediatric and young adult patients. Pediatr Radiol. 2012;42(12):1437-1440.

22. El-Serag HB, Sweet $S$, Winchester CC, et al. Update on the epidemiology of gastro-oesophageal reflux disease: a systematic review. Gut. 2014;63(6):871-880.

23. Eluri S, Dellon ES. Proton pump inhibitor-responsive oesophageal eosinophilia and eosinophilic oesophagitis: more similarities than differences. Curr Opin Gastroenterol. 2015;31(4):309-315.

\section{Figures}


Total number of cases identified $(n=545)$

- ICD-9 and/or ICD-10 diagnosis of eosinophilic esophagitis (EOE): Including visit diagnosis, billing diagnosis, problem list or past medical history of EOE

\section{Pediatric cases removed} (age at diagnosis less than 18 years $)(n=44)$

\section{Non-EOE cases excluded $(n=210)$}

- Esophageal eosinophil count less than 15/High power field

- Lack of esophageal symptoms $^{\star \star}$

\section{Total cases included for EMR review* $(n=501)$}

Total eosinophilic esophagitis (EOE) cases included in analysis $(n=291)$

Figure 1

Flowchart of selection criteria for EoE cases. *EMR: Electronic Medical Record

**Esophageal symptoms include dysphagia, odynophagia, food impaction, chest pain, epigastric pain, nausea, vomiting, heartburn and regurgitation. 


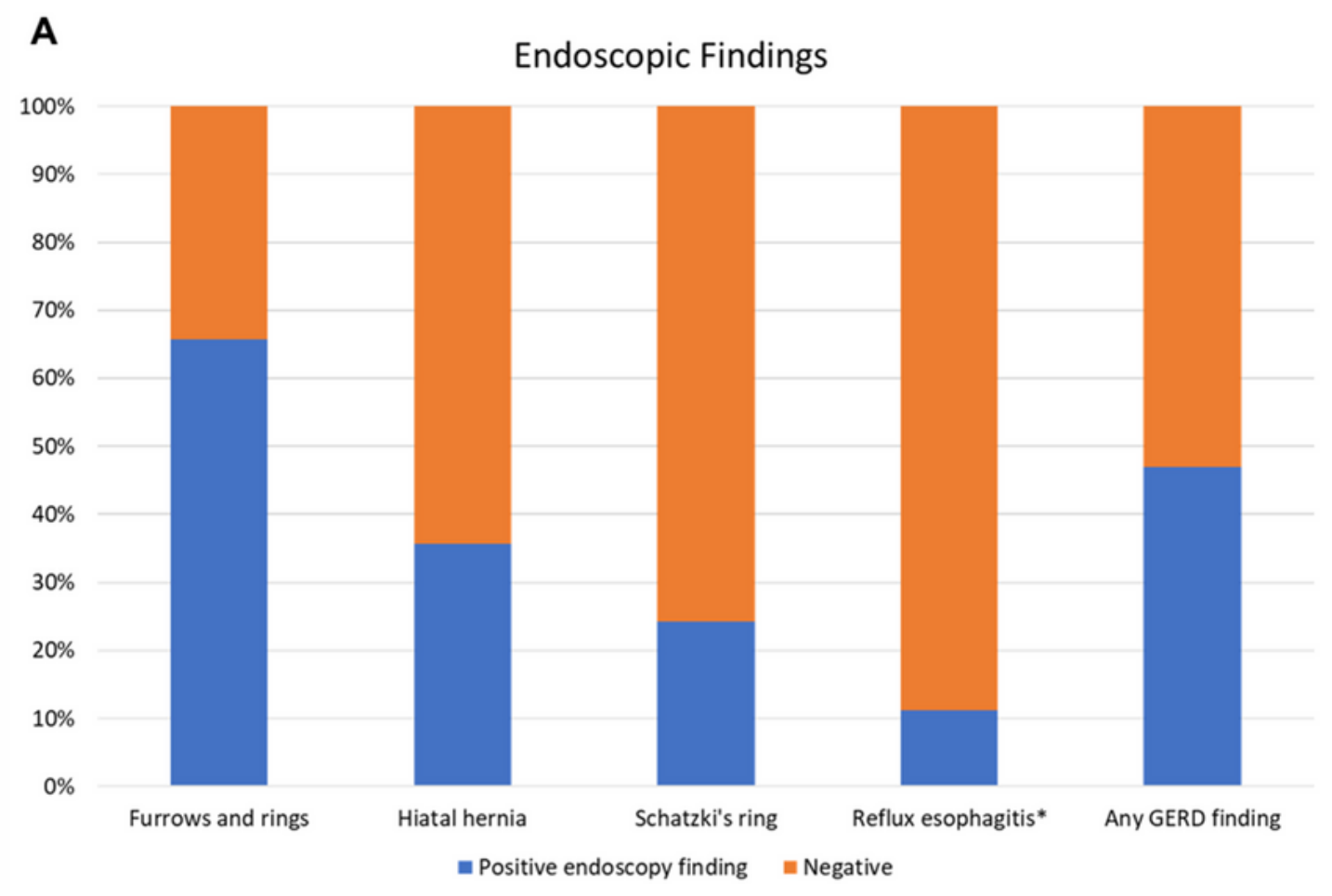

B

$P=.045$

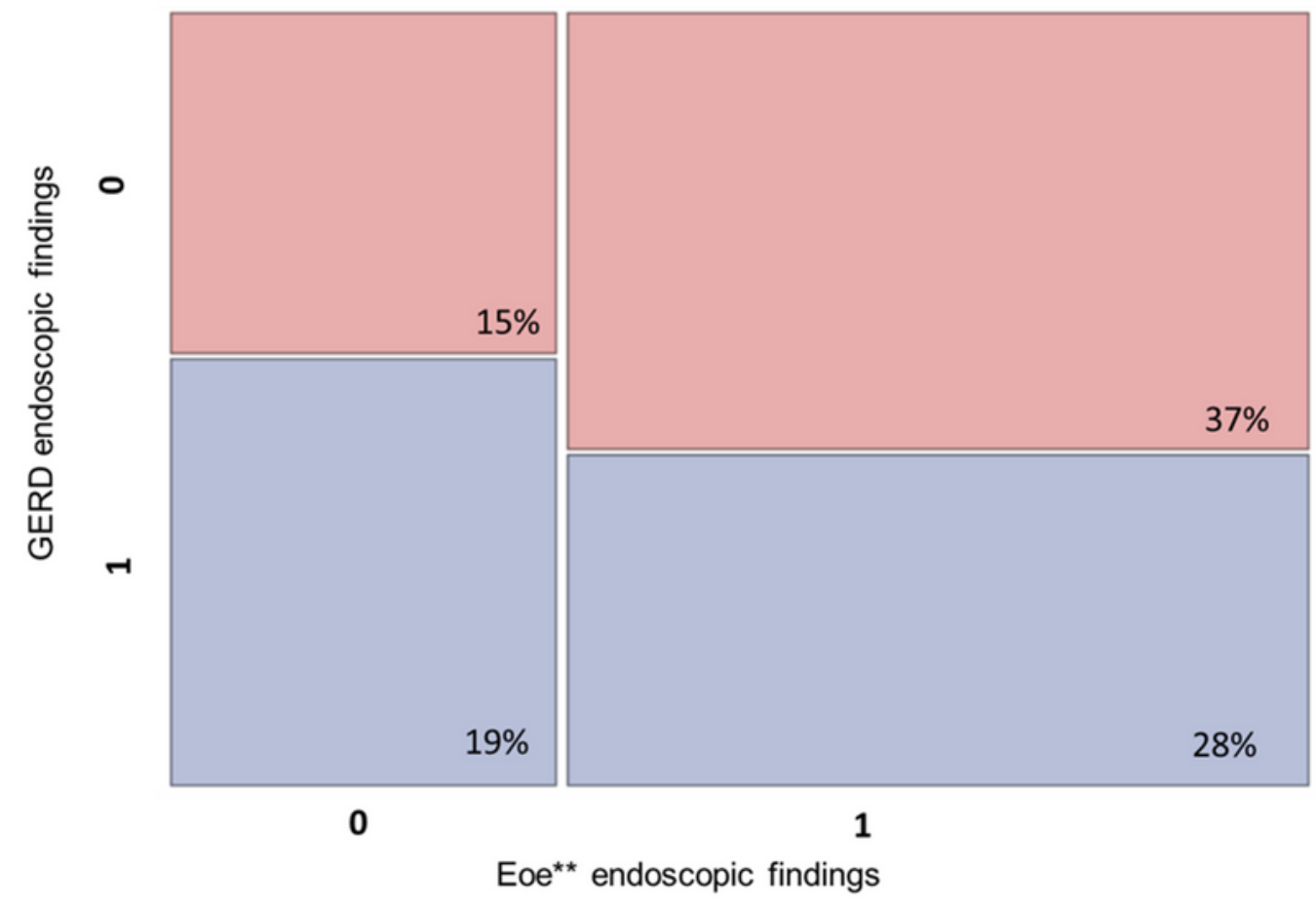

Figure 2

Endoscopic findings of eosinophilic esophagitis cases. A. Percentage of patients who had classic EoE endoscopic feature (longitudinal furrows and/or concentric rings); and different findings suggestive of gastroesophageal reflux disease (GERD). B. Mosaic plot of endoscopic findings in EoE patients showing different combinations of EoE and GERD findings. 
*Reflux esophagitis: LA Class B esophagitis or greater noted on endoscopy, "any GERD finding" indicate the percentage of patients who had hiatal hernia, Schatzki's ring or reflux esophagitis. ${ }^{\star}$ EoE: eosinophilic esophagitis.

A Food impaction in patients with EOE endoscopic findings vs no EOE endoscopic findings
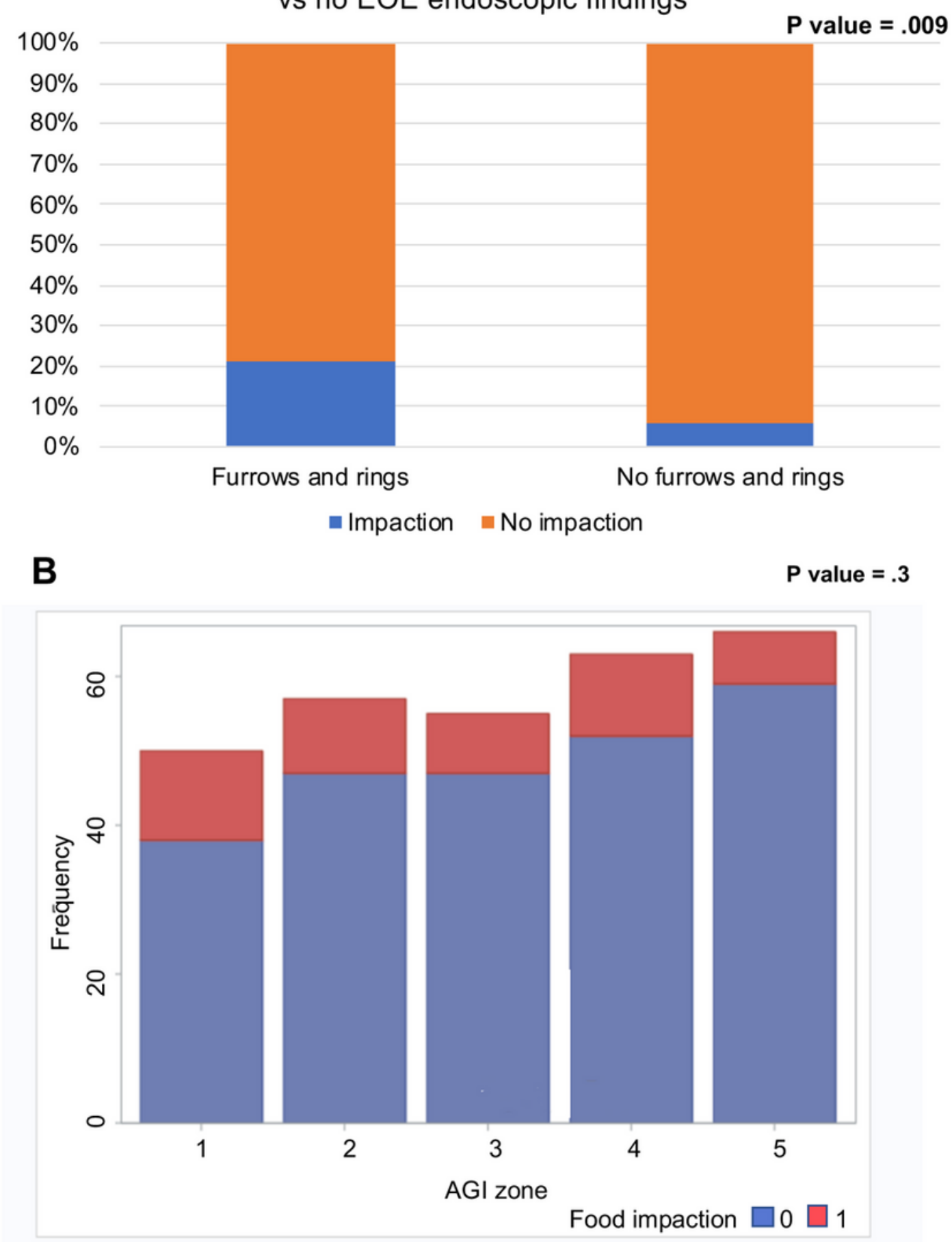

Figure 3 
Esophageal food impaction outcome. A. Percentage of food impactions based on the presence of classic eosinophilic esophagitis endoscopic findings. B. Frequency of food impaction based on socioeconomic status. AGI: Adjusted Gross Income

\section{Supplementary Files}

This is a list of supplementary files associated with this preprint. Click to download.

- RawData.xlsx

- SupplementaryFigure1.png

- Supplementaryfigure1legend.docx 